\title{
Symmetric Plasmas and Their Decay
}

\section{E. Horst *}

FB Mathematik der GHS, Warburger Straße 100, D-4790 Paderborn,

Federal Republic of Germany

\begin{abstract}
Spherically symmetric global solutions are shown to exist for the relativistic Vlasov-Maxwell system of plasma physics. In view of a conjectured perturbation result concerning in particular "nearly" symmetric global solutions, we investigate the asymptotic properties of the symmetric solutions. In the case of only one particle species (say ions but no electrons) we get satisfactory decay estimates; in the general case (ions and electrons) we have preliminary results.
\end{abstract}

\section{Introduction}

The relativistic Vlasov-Maxwell system (RVMS) consists of Vlasov's equations for the ion distribution $f^{+}$and the electron distribution $f^{-}$

$$
\partial_{t} f^{ \pm}+\hat{u} \partial_{x} f^{ \pm} \pm(E(t, x)+d \hat{u} \wedge B(t, x)) \partial_{u} f^{ \pm}=0,
$$

Maxwell's equations for the electric and magnetic fields

$$
\begin{gathered}
d \partial_{t} E=\operatorname{curl}_{x} B-d \gamma 4 \pi j(t, x), \\
d \partial_{t} B=-\operatorname{curl}_{x} E, \\
\operatorname{div}_{x} E=\gamma 4 \pi \varrho(t, x), \\
\operatorname{div}_{x} B=0,
\end{gathered}
$$

and the equations for charge and current that couple Vlasov's and Maxwell's equations

$$
\begin{gathered}
j(t, x)=\int \hat{u}\left(f^{+}(t, x, u)-f^{-}(t, x, u)\right) \mathrm{d} u, \\
\varrho(t, x)=\int\left(f^{+}(t, x, u)-f^{-}(t, x, u)\right) \mathrm{d} u .
\end{gathered}
$$

\footnotetext{
* Parts of this paper were written during a stay at Indiana University, Bloomington. The author gratefully acknowledges many pleasant conversations with R. Glassey
} 
Here $d \geqq 0$ denotes the inverse of the speed of light, $d=c_{0}^{-1}$, the ion and electron densities $f^{+}(t, x, u)$ and $f^{-}(t, x, u)$ are functions of time $t \geqq 0$, position $x \in \mathbb{R}^{3}$, and momentum $u \in \mathbb{R}^{3}$. The relativistic velocity $\hat{u}$ is given by $\hat{u}=\left(1+d^{2} u^{2}\right)^{-1 / 2} u . E(t, x)$ and $B(t, x)$ are the electric and magnetic fields; we assume that for fixed time $t$ these fields are square-integrable over $\mathbb{R}^{3} . \varrho(t, x)$ and $j(t, x)$ are the charge and current densities, respectively.

If the parameter $\gamma$ is chosen as $\gamma=+1$, the equations describe a plasma, while for $\gamma=-1$ they model the evolution of a galaxy.

In the present paper we consider the initial-value problem and, therefore, we prescribe initial data $f_{0}^{ \pm}=f^{ \pm}(0, \cdot), E_{0}=E(0, \cdot), B_{0}=B(0, \cdot)$. We assume that Eqs. (4) and (5) are satisfied for $t=0$ and that $f_{0}^{+}$and $f_{0}^{-}$are continuously differentiable, nonnegative and have compact support. We are only interested in global (-in-time) solutions of the equations, since we study their asymptotic properties.

If $\hat{u}$ is replaced by $u$ wherever it appears, the resulting system is called the Vlasov-Maxwell System (VMS). If $d$ is replaced by zero in Maxwell's equations (and only there), we get the relativistic Vlasov-Poisson System (RVPS). If $d=0$ in all equations (which implies $u=\hat{u}$ ), we get the Vlasov-Poisson System (VPS).

If $d=0$, Maxwell's equations degenerate into the following system:

$$
\operatorname{curl}_{x} B=0, \quad \operatorname{curl}_{x} E=0, \quad \operatorname{div}_{x} E=\gamma 4 \pi \varrho, \quad \operatorname{div}_{x} B=0 .
$$

Since $\operatorname{curl}_{x} E(t, x)=0, E(t, \cdot)$ is a gradient field, $E(t, x)=-\operatorname{grad}_{x} U(t, x) . U$ satisfies Poisson's equation

and hence we have

$$
\Delta_{x} U=-\gamma 4 \pi \varrho
$$

$$
U(t, x)=\gamma \int \frac{1}{|x-y|} \varrho(y) \mathrm{d} u
$$

(plus a harmonic function that must be constant in order to achieve the squareintegrability of $E(t, \cdot)=-\operatorname{grad} U(t, \cdot))$, which in turn implies

$$
E(t, x)=\gamma \int \frac{x-y}{|x-y|^{3}} \varrho(t, y) \mathrm{d} y .
$$

An analogous argument proves that

$$
B(t, x) \equiv 0 \text {. }
$$

Thus in the degenerate case $d=0$, Maxwell's equations can be replaced by Eqs. (8) and (9). In this situation no initial data can be prescribed for $E$ and $B: B(0, \cdot)=0$ and $E(0, \cdot)$ is computed from $f_{0}^{ \pm}$.

During the last dozen years much work has been done on these equations, but usually the authors considered only special choices of the parameters $d$ and $\gamma$. We use a unified approach that covers all cases simultaneously. This is reflected in the following definition of a solution of RVMS etc., which is easy to handle but perhaps more restrictive than necessary.

(0.1) Definition. (a) We say that continuously differentiable functions $f^{+}$, $f^{-}:\left[0, \infty\left[\times \mathbb{R}^{3} \times \mathbb{R}^{3} \rightarrow\left[0, \infty\left[; E, B:\left[0, \infty\left[\times \mathbb{R}^{3} \rightarrow \mathbb{R}^{3}\right.\right.\right.\right.\right.\right.$ constitute a solution of RVMS, if 
(i) For every $T \geqq 0$ there exists a compact subset of $\mathbb{R}^{3} \times \mathbb{R}^{3}$ containing the supports of $f^{+}(t, \cdot)$ and $f^{-}(t, \cdot)$ for all $t \in[0, T]$.

(ii) The differential equations (1)-(5) are satisfied everywhere with $\varrho$ and $j$ being defined by (6) and (7). (The integrations involved in the definition of $\varrho$ and $j$ pose no problems because of (i).)

(iii) For every $T \geqq 0$ the fields $E$ and $B$ together with all their derivatives are uniformly bounded on $[0, T] \times \mathbb{R}^{3}$.

(iv) $E(t, \cdot)$ and $B(t, \cdot)$ are square integrable for all $t \geqq 0$.

(b) A solution of VMS, RVPS or VPS is defined the same way, but with $d$ replaced by zero in the appropriate places.

In order to fix notation we describe how the method of characteristics is applied to the construction of the solutions $f^{+}$and $f^{-}$of Vlasov's equations (cf. [8]): Consider the system of ordinary differential equations

$$
\begin{aligned}
& \dot{X}^{ \pm}=\hat{U}^{ \pm}, \\
& \dot{U}^{ \pm}= \pm\left(E\left(t, X^{ \pm}\right)+d \hat{U}^{ \pm} \wedge B\left(t, X^{ \pm}\right)\right) .
\end{aligned}
$$

For every $\tau \geqq 0$ and every pair $(x, u) \in \mathbb{R}^{3} \times \mathbb{R}^{3}$ we denote by $X^{ \pm}(t, \tau, x, u)$, $U^{ \pm}(t, \tau, x, u)$ the solution of $(10)$ with $\left(X^{ \pm}(\tau, \tau, x, u), U^{ \pm}(\tau, \tau, x, u)\right)=(x, u)$. With this definition we have

$$
f^{ \pm}(t, x, u)=f_{0}^{ \pm}\left(X^{ \pm}(0, t, x, u), U^{ \pm}(0, t, x, u)\right) .
$$

Moreover, for fixed $t$ the mapping $\mathbb{R}^{6} \rightarrow \mathbb{R}^{6}$,

$$
(x, u) \mapsto\left(X^{ \pm}(0, t, x, u), U^{ \pm}(0, t, x, u)\right)
$$

is a diffeomorphism with determinant of the Jacobian equal to unity (which is extremely useful for computing integrals). The inverse of the mapping (11) is given by

$$
(x, u) \mapsto\left(X^{ \pm}(t, 0, x, u), U^{ \pm}(t, 0, x, u)\right) .
$$

Using the substitution (11) we get that all $L^{p}$-norms $(1 \leqq p \leqq \infty)$ of $f^{ \pm}(t, \cdot)$ are independent of $t \in \mathbb{R}$. If $p=1$, this is conservation of total charge.

An important tool in some of the known existence results is conservation of energy. This means that the total energy

$$
H:=\int \frac{u^{2}}{\sqrt{1+d^{2} u^{2}}+1}\left(f^{+}(t, x, u)+f^{-}(t, x, u)\right) \mathrm{d} x \mathrm{~d} u+\frac{1}{8 \pi \gamma} \int\left(E(t, x)^{2}+B(t, x)^{2}\right) \mathrm{d} x
$$

is conserved. The first term is the kinetic energy and the second one the field energy. If $\gamma=+1$, both energies are nonnegative and an a-priori estimate is obtained easily. (But energy also has its uses if $\gamma=-1, d=0$, cf. [7].)

Recent work by Schaeffer and others (cf. $[12,3,5,11]$ ) suggests the following

Conjecture. Assume that $\left(f^{+}, f^{-}, E, B\right)$ is a solution of $R V M S$ such that for all $(x, u) \in \operatorname{supp} f^{+}(t, \cdot) \cup \operatorname{supp} f^{-}(t, \cdot)$,

$$
\begin{gathered}
|E(t, x)|,|B(t, x)| \leqq g(t), \\
\left|\partial_{x} E(t, x)\right|,\left|\partial_{x} B(t, x)\right| \leqq h(t)
\end{gathered}
$$


with continuous decreasing functions $g, h:[0, \infty[\rightarrow[0, \infty[$ satisfying

$$
\int_{0}^{\infty}(g(t)+t h(t)) \mathrm{d} t<\infty .
$$

Then a global solution of RVMS exists for all initial data near (in some suitable norm) $\left(f_{0}^{+}, f_{0}^{-}, E_{0}, B_{0}\right)$.

Moreover, the perturbed solution satisfies estimates similar to (12) and (13) (and many more that follow from them).

In the present paper we are not concerned with proving the conjecture, but we prove global existence for a class of symmetric solutions of RVMS with $B \equiv 0$ and investigate their behaviour for large times. In the case that only ions are present $\left(f_{0}^{-} \equiv 0\right)$ we can show decay like

$$
\begin{aligned}
|E(t, x)| & \leqq \frac{C}{(1+t)^{2}} \\
\left|\partial_{x} E(t, x)\right| & \leqq C \frac{(1+\log (1+t))^{3}}{(1+t)^{3}} .
\end{aligned}
$$

If, however, $f_{0}^{-} \neq 0$, we have only preliminary results. (Of course, the conjecture implies that there is still decay if $f_{0}^{-} \neq 0$ but is small enough.)

\section{Additional Remarks}

(i) In [4] we investigated a related but different problem for RVMS with $d \neq 0$. The initial conditions for $E$ and $B$ were replaced by the condition that the field energy tend to zero as $t \rightarrow \infty$. Numerous pre-1986 references can be found in this paper.

(ii) We do not consider weak solutions of RVMS (cf. e.g. [1] and [9]), since an estimate like (12) guarantees the existence of classical solutions anyway (cf. [4] and [6]).

(iii) Our treatment of the cases $d=0$ and $d>0$ at the same time may look somewhat artificial. It is, however, economical to treat VPS as a borderline case of RVMS, since many results for the case $d>0$ (but not all) remain true for $d=0$. Moreover, as we will soon see, a symmetric solution of RVPS is also a solution of RVMS. Since so far the Poisson case is much better understood than the Maxwell case, this is a very helpful observation.

(iv) If $\gamma=-1$, the equations describe models from stellar dynamics. Since there is only one type of mass (positive) in contrast to two types of electric charge (positive and negative), we may assume $f_{0}^{-}=0$ in this case.

\section{Symmetry and Global Existence}

(1.1) Definition and Lemma. Consider continuous functions

$$
\begin{gathered}
\varphi: \mathbb{R}^{3} \times \mathbb{R}^{3} \rightarrow \mathbb{R}, \\
\sigma: \mathbb{R}^{3} \rightarrow \mathbb{R}, \\
K: \mathbb{R}^{3} \rightarrow \mathbb{R}^{3} .
\end{gathered}
$$


We say that $\varphi, \sigma$ or $K$, respectively, is spherically symmetric, if for all proper rotations $R$ of $\mathbb{R}^{3}$ (equivalently for all rotations) and all $x, u \in \mathbb{R}^{3}$,

$$
\varphi(R x, R u)=\varphi(x, u), \quad \sigma(R x)=\sigma(x), \quad K(R x)=R K(x),
$$

respectively.

The functions, $\varphi, \sigma, K$ are spherically symmetric, if and only if there exist scalar functions $\tilde{\varphi}, \tilde{\sigma}, \tilde{K}$, respectively, such that

respectively.

$$
\begin{gathered}
\varphi(x, u)=\tilde{\varphi}\left(x^{2}, x u, u^{2}\right), \\
\sigma(x)=\tilde{\sigma}(|x|), \\
K(x)=x \widetilde{K}(|x|),
\end{gathered}
$$

Proof. Assume that $\varphi, \sigma$, and $K$ are invariant under proper rotations. (14) follows from the fact that, given $x, u, y, w \in \mathbb{R}^{3}$ with $|x|=|y|,|u|=|w|, x u=y w$, there exists a proper rotation $R$ with $y=R x, w=R u$.

Equation (15) is trivial. Equation (16) follows e.g. from the Hedgehog (aka Haired-Sphere) Theorem (a continuous tangential field on a sphere must vanish somewhere): For any $r>0$ there exists a point on the sphere $|x|=r$ where the field $K$ is normal and, because of the symmetry, $K$ is therefore normal everywhere on the sphere.

Since the conditions (14), (15), and (16) are invariant under all rotations, it is clear that we do not have to restrict $R$ to the group of proper rotations.

We remark that, if $\varphi$ is spherically symmetric, there exists a function $\bar{\varphi}$ such that for all $x, u \in \mathbb{R}^{3}, x \neq 0$ we have that $\varphi(x, u)=\bar{\varphi}\left(|x|,|x|^{-1} x u, x^{2} u^{2}-(x u)^{2}\right)$. This choice of coordinates will be quite convenient in a later calculation. The reason is the following

(1.2) Lemma (Integration Lemma). Assume that $\bar{\varphi}:] 0, \infty[\times \mathbb{R} \times[0, \infty[\rightarrow[0, \infty[$ is measurable and that $\varphi(x, u)=\bar{\varphi}\left(|x|,|x|^{-1} x u, x^{2} u^{2}-(x u)^{2}\right)$ for all $x, u \in \mathbb{R}^{3}, x \neq 0$. Then

$$
\int \varphi(x, u) \mathrm{d} x \mathrm{~d} u=4 \pi^{2} \int_{0}^{\infty} \int_{-\infty}^{\infty} \int_{0}^{\infty} \bar{\varphi}(a, b, c) \mathrm{d} a \mathrm{~d} b \mathrm{~d} c .
$$

Proof. Let $x$ be fixed. We first integrate $\varphi(x, u)$ over $u \in \mathbb{R}^{3}$. Rotating $u$-space, if necessary, we may assume that $x$ is a multiple of the first unit vector. Thus

$$
\int \varphi(x, u) \mathrm{d} u=\iiint \bar{\varphi}\left(|x|, u_{1}, x^{2}\left(u_{2}^{2}+u_{3}^{2}\right)\right) \mathrm{d} u_{1} \mathrm{~d} u_{2} \mathrm{~d} u_{3} .
$$

Now we rename $u_{1}$, calling it $b$, and use polar coordinates for the $\left(u_{2}, u_{3}\right)$-integral. This gives

$$
\int \varphi(x, u) \mathrm{d} u=2 \pi \int_{-\infty}^{\infty} \int_{0}^{\infty} r \bar{\varphi}\left(|x|, b, x^{2} r^{2}\right) \mathrm{d} r \mathrm{~d} b=\frac{\pi}{x^{2}} \int_{-\infty}^{\infty} \int_{0}^{\infty} \bar{\varphi}(|x|, b, c) \mathrm{d} c \mathrm{~d} b,
$$

if we substitute $c=x^{2} r^{2}$. Integration over $x \in \mathbb{R}^{3}$ now yields the claimed result.

The importance of spherical symmetry for the present investigation is that it is preserved in time, i.e., if the initial data are symmetric, then the solution will remain symmetric. Moreover, global existence poses no problem in the plasma case with symmetry: 
(1.3) Theorem. Assume that $f_{0}^{+}$and $f_{0}^{-}$are spherically symmetric, nonnegative, continuously differentiable and have compact support. If $\gamma=+1$, there exists a unique global solution of RVPS. It has the following properties:

(i) $f^{ \pm}(t, \cdot), \varrho(t, \cdot), j(t, \cdot), E(t, \cdot)$ are spherically symmetric for all $t \geqq 0$; more specifically we have

$$
E(t, x)=\frac{x}{|x|^{3}} \int_{|y| \leqq|x|} \varrho(t, y) \mathrm{d} y
$$

for all $t \geqq 0, x \in \mathbb{R}^{3}$.

(ii) (Conservation of Angular Momentum): For all $t, \tau \geqq 0, x, u \in \mathbb{R}^{3}$,

$$
X^{ \pm}(t, \tau, x, u) \wedge U^{ \pm}(t, \tau, x, u)=x \wedge u .
$$

(iii) There exists a constant $C$, such that for all $t, \tau \geqq 0 ; x, u \in \mathbb{R}^{3}$,

$$
\begin{gathered}
|| U^{ \pm}(t, \tau, x, u)|-| u|| \leqq C, \\
|\varrho(t, x)| \leqq C, \\
|j(t, x)| \leqq C, \\
|E(t, x)| \leqq C, \\
\left|\partial_{x} E(t, x)\right| \leqq C, \\
f^{ \pm}(t, x, u)=0, \text { if }|u| \geqq C .
\end{gathered}
$$

Sketch of the proof. We will not give a complete proof of this result, since this proof is easily carried out, using the Frankenstein method. Nearly all parts of the argument are already available, but they have to be assembled in a different manner to form a new entity. If $d=0$, the proof is exactly the same as that of Theorem (7.3) of [7]. This is a result for a single distribution function, but can be generalized to a system immediately. The case $d>0$ with only a single distribution function was investigated in [2]. In order to generalize Theorem II of this paper to the case of a system, it is only necessary to find a substitute for Lemma IV and Lemma $\mathrm{V}$ of [6]. This will be the purpose of our next lemma. But we want to clarify the following point: Theorem II of [2] contains the assumption that $f_{0}(x, u)$ vanishes if the angular momentum $x \wedge u$ vanishes. This assumption is only an artefact due to the choice of coordinate system. If the computations are carried out in cartesian coordinates as in [7], this becomes immediately evident.

The statement of the theorem contains two claims that cannot be found in [2], nor in [7]. The spherical symmetry of $j(t, \cdot)$ follows at once from the spherical symmetry of $f^{ \pm}(t, \cdot)$ and integration. The estimate (22) follows from (19) and Lemma (2.2), infra.

(1.4) Lemma. Assume that $T>0$ and that $E:[0, T] \times \mathbb{R}^{3} \rightarrow \mathbb{R}^{3}$ is a continuous function of the form $E(t, x)=x \widetilde{E}(t, x)$ with a scalar function $\widetilde{E}$. Assume that $E$ satisfies an estimate

$$
|E(t, x)| \leqq g(|x|)
$$

with $g:\left[0, \infty\left[\rightarrow\left[0, \infty\left[\right.\right.\right.\right.$ decreasing and $\|g\|_{1}:=\int_{0}^{\infty} g(r) \mathrm{d} r<\infty$. 
Assume further that the functions $X, U:[0, T] \rightarrow \mathbb{R}^{3}$ are continuously differentiable and satisfy the differential equation

$$
\begin{gathered}
\frac{\mathrm{d}}{\mathrm{d} t} X=\hat{U}:=\left(1+d^{2} U^{2}\right)^{-1 / 2} U, \\
\frac{\mathrm{d}}{\mathrm{d} t} U=E(t, X),
\end{gathered}
$$

with some fixed $d>0$. Then we have

$$
\left|\sqrt{1+d^{2}|U(T)|^{2}}-\sqrt{1+d^{2}|U(0)|^{2}}\right| \leqq 3 d^{2}\|g\|_{1} .
$$

Remark. This lemma is completely independent from the rest of the paper, but we use a suggestive notation. Thus the letters $X, U, E$, and $d$ have a similar meaning when the lemma is applied in the RVMS situation.

Proof. $1^{\circ}$ Without loss of generality we may assume $d=1$, the case $d \neq 1$ can be proved by applying this case to $d X$ and $d U$ instead of $X$ and $U$. Moreover, we may assume $\|g\|_{1}>0$, since otherwise $U$ is constant and (24) is trivial.

$2^{\circ}$ Angular momentum is conserved:

$$
\frac{\mathrm{d}}{\mathrm{d} t}(X \wedge U)=\hat{U} \wedge U+X \wedge E(t, X)=0
$$

In particular, the square of $X \wedge U$ is a constant:

$$
|X \wedge U|^{2}=X^{2} U^{2}-\langle X, U\rangle^{2}=: c=\text { const . }
$$

(We sometimes write $\langle\cdot, \cdot\rangle$ for the scalar product of $\mathbb{R}^{3}$.)

$3^{\circ}$ Now we assume that $[\tau, \vartheta]$ is a subinterval of $[0, T]$ and that $\langle X, U\rangle$ has no zero in $] \tau, \vartheta[$. Let us call this the simple case for $[\tau, \vartheta]$. Then

$$
\begin{aligned}
& \left|\sqrt{1+|U(\vartheta)|^{2}}-\sqrt{1+|U(\tau)|^{2}}\right|=\left|\int_{\tau}^{\vartheta} \frac{\mathrm{d}}{\mathrm{d} s} \sqrt{1+|U(s)|^{2}} \mathrm{~d} s\right| \\
& =\left|\int_{\tau}^{\vartheta}\langle\hat{U}(s), E(s, X(s))\rangle \mathrm{d} s\right|=\left|\int_{\tau}^{\vartheta}\left\langle\hat{U}(s), \frac{X(s)}{|X(s)|}\right\rangle\right| X(s)|\tilde{E}(s, X(s)) \mathrm{d} s| \\
& \leqq\left|\int_{\tau}^{\vartheta}\left\langle\hat{U}(s), \frac{X(s)}{|X(s)|}\right\rangle g(|X(s)|) \mathrm{d} s\right|
\end{aligned}
$$

(Remember: $\langle U, X\rangle$ does not change sign in $[\tau, \vartheta]$.)

$$
=\left|\int_{\tau}^{\vartheta}\left(\frac{\mathrm{d}}{\mathrm{d} s}|X(s)|\right) g(|X(s)|) \mathrm{d} s\right|=\left|\int_{|X(\tau)|}^{|X(9)|} g(r) \mathrm{d} r\right| .
$$

This shows that in the simple case for $[\tau, \vartheta]$,

$$
\left|\sqrt{1+|U(\vartheta)|^{2}}-\sqrt{1+|U(\tau)|^{2}}\right| \leqq\left|\int_{|X(\tau)|}^{|X(\vartheta)|} g(r) \mathrm{d} r\right| .
$$

$4^{\circ}$ If $\langle X, U\rangle$ has no zero in $] 0, T[$, then (24) follows immediately from the simple case for $[0, T]$. 
Let us now consider the case that $\langle X, U\rangle$ does have zeroes in $] 0, T[$. For the time being we assume $c \neq 0$. This implies $X(t) \neq 0$ and $U(t) \neq 0$ for all $t \in[0, T]$. We will only show that

$$
\sqrt{1+|U(T)|^{2}} \leqq \sqrt{1+|U(0)|^{2}}+3\|g\|_{1},
$$

the other half of (24) is proved with the same method, but "going backward in time."

The set

$$
Z:=\{t \in] 0, T[\mid\langle X(t), U(t)\rangle=0\}
$$

is not empty. Let $t_{-}=\inf Z, t_{+}=\sup Z$. Then $t_{ \pm} \in Z \cup\{0, T\}$ and $\left\langle X\left(t_{ \pm}\right), U\left(t_{ \pm}\right)\right\rangle=0$. The continuous function $|X(t)|^{2}$ attains its maximum at some point $t_{0}$ of the compact interval $\left[t_{-}, t_{+}\right]$. Clearly $\left\langle X\left(t_{0}\right), U\left(t_{0}\right)\right\rangle=0$. (If $\left.t_{0} \in\right] t_{-}, t_{+}\left[\right.$, this follows from $\left.\frac{\mathrm{d}}{\mathrm{d} t} X^{2}\right|_{t=t_{0}}=0$, if $t_{0} \in\left\{t_{-}, t_{+}\right\}$, we already know it.)

We first claim that for all $t \in\left[t_{0}, t_{+}\right]$,

$$
\sqrt{1+|U(t)|^{2}} \leqq \sqrt{1+|U(0)|^{2}}+\|g\|_{1}+\int_{|X(t)|}^{\left|X\left(t_{0}\right)\right|} g(s) \mathrm{d} s .
$$

In order to prove this we will show that the set

$$
S:=\left\{s \in\left[t_{0}, t_{+}\right] \mid(25) \text { is true for all } t \in\left[t_{0}, s\right]\right\}
$$

is nonempty, and open and closed in the interval $\left[t_{0}, t_{+}\right]$. This implies $S=\left[t_{0}, t_{+}\right]$. $S$ is nonempty, since $t_{0} \in S$ : We have

$$
\begin{aligned}
\sqrt{1+\left|U\left(t_{0}\right)\right|^{2}} & =\sqrt{1+\frac{c}{\left|X\left(t_{0}\right)\right|^{2}}} \leqq \sqrt{1+\frac{c}{\left|X\left(t_{-}\right)\right|^{2}}}=\sqrt{1+\left|U\left(t_{-}\right)\right|^{2}} \\
& \leqq \sqrt{1+|U(0)|^{2}}+\|g\|_{1}=\sqrt{1+|U(0)|^{2}}+\|g\|_{1}+\int_{\left|X\left(t_{0}\right)\right|}^{\left|X\left(t_{0}\right)\right|} g(r) \mathrm{d} r .
\end{aligned}
$$

(If $t_{-}=0$, this is trivial, otherwise it follows from the simple case for the interval $\left.\left[0, t_{-}\right].\right)$

$S$ is clearly closed in $\left[t_{0}, t_{+}\right]$. To show that $S$ is open in $\left[t_{0}, t_{+}\right]$assume $t \in S$, $t<t_{+}$. We consider the three possible cases.

First Case. There exists $\varepsilon>0, \varepsilon \leqq t_{+}-t$, such that

$$
\left.\langle X, U\rangle\right|_{] t, t+\varepsilon[}<0 \text {. }
$$

( $X^{2}$ is decreasing to the right of $t$; this is the easy case.) Assume $\left.s \in\right] t, t+\varepsilon[$. Then, using the fact that $t \in S$ and the simple case for $[t, s]$, we get that

$$
\begin{aligned}
\sqrt{1+|U(s)|^{2}} & \leqq \sqrt{1+|U(t)|^{2}}+\int_{|X(s)|}^{|X(t)|} g(r) \mathrm{d} r \\
& \leqq \sqrt{1+|U(0)|^{2}}+\|g\|_{1}+\int_{|X(t)|}^{\left|X\left(t_{0}\right)\right|} g(r) \mathrm{d} r+\int_{|X(s)|}^{|X(t)|} g(r) \mathrm{d} r \\
& =\sqrt{1+|U(0)|^{2}}+\|g\|_{1}+\int_{|X(s)|}^{\left|X\left(t_{0}\right)\right|} g(r) \mathrm{d} r .
\end{aligned}
$$

This shows $[t, t+\varepsilon[C S$. 
Second Case. There exists $\varepsilon>0, \varepsilon \leqq t_{+}-t$ such that

$$
\left.\langle X, U\rangle\right|_{], t+\varepsilon[}>0 \text {. }
$$

( $X^{2}$ is increasing to the right of $t$; this is the hard case.) Assume $\left.s \in\right] t, t+\varepsilon[$. Let

$$
s_{+}:=\inf \left(\left(Z \cup\left\{t_{+}\right\}\right) \cap\left[t+\varepsilon, t_{+}\right]\right) \geqq t+\varepsilon>s .
$$

Thus $s_{+}$is the smallest zero of $\langle X, U\rangle=\frac{1}{2} \sqrt{1+U^{2}} \frac{\mathrm{d}}{\mathrm{d} t} X^{2}$ to the right of $t+\varepsilon$. Since $X^{2}$ is strictly increasing on $\left[t, s_{+}\right]$, we have that

$$
|X(t)|<|X(s)|<\left|X\left(s_{+}\right)\right| .
$$

Since $\left|X\left(t_{0}\right)\right| \geqq\left|X\left(s_{+}\right)\right|$and $|X(t)|<\left|X\left(s_{+}\right)\right|$, there exists (Intermediate-Value Theorem!) an $s_{-} \in\left[t_{0}, t[\right.$ such that

$$
\left|X\left(s_{-}\right)\right|=\left|X\left(s_{+}\right)\right| .
$$

Using the simple case for $\left[s, s_{+}\right]$we get

$$
\begin{aligned}
\sqrt{1+|U(s)|^{2}} & \leqq \sqrt{1+\left|U\left(s_{+}\right)\right|^{2}}+\int_{|X(s)|}^{\left|X\left(s_{+}\right)\right|} g(r) \mathrm{d} r \\
& =\sqrt{1+\frac{c}{\left|X\left(s_{+}\right)\right|^{2}}}+\int_{|X(s)|}^{\left|X\left(s_{+}\right)\right|} g(r) \mathrm{d} r \\
& =\sqrt{1+\frac{c}{\left|X\left(s_{-}\right)\right|^{2}}}+\int_{|X(s)|}^{\left|X\left(s_{-}\right)\right|} g(r) \mathrm{d} r \\
& \leqq \sqrt{1+\frac{c+\left\langle X\left(s_{-}\right), U\left(s_{-}\right)\right\rangle^{2}}{\left|X\left(s_{-}\right)\right|^{2}}}+\int_{|X(s)|}^{\left|X\left(s_{-}\right)\right|} g(r) \mathrm{d} r \\
& =\sqrt{1+\left|U\left(s_{-}\right)\right|^{2}}+\int_{|X(s)|}^{\left|X\left(s_{-}\right)\right|} g(r) \mathrm{d} r \\
& \leqq \sqrt{1+|U(0)|^{2}}+\|g\|_{1}+\int_{\left|X\left(s_{-}\right)\right|}^{\left|X\left(t_{0}\right)\right|} g(r) \mathrm{d} r+\int_{|X(s)|}^{\left|X\left(s_{-}\right)\right|} g(r) \mathrm{d} r \\
& =\sqrt{1+|U(0)|^{2}}+\|g\|_{1}+\int_{|X(s)|}^{\left|X\left(t_{0}\right)\right|} g(r) \mathrm{d} r .
\end{aligned}
$$

[Here we have used the fact that $s_{-}<t$ and therefore (25) is true for $s_{-}$. ] Again this shows $[t, t+\varepsilon[C S$.

Third Case. For every $\delta>0$ the function $\langle X, U\rangle$ changes sign on $] t, t+\delta[$. Then there exists a sequence $\left.\left.\left(t_{n}\right) \subset\right] t, t_{+}\right]$with $\langle X, U\rangle\left(t_{n}\right)=0$ and $t_{n} \rightarrow t$. Rolle's Theorem implies that there exists a sequence $\left.\left.\left(s_{n}\right) \subset\right] t, t_{+}\right]$with $\langle X, U\rangle^{\prime}\left(s_{n}\right)=0$ and $s_{n} \rightarrow t$. Thus

$$
\begin{aligned}
0=\langle X, U\rangle^{\prime}(t) & =\frac{|U(t)|^{2}}{\sqrt{1+|U(t)|^{2}}}+|X(t)|^{2} \widetilde{E}(t, x(t)) \\
& \geqq \frac{|U(t)|^{2}}{\sqrt{1+|U(t)|^{2}}}-|X(t)| g(|X(t)|) .
\end{aligned}
$$


This shows that

$$
\begin{aligned}
\sqrt{1+|U(t)|^{2}} & =\frac{1+|U(t)|^{2}}{\sqrt{1+|U(t)|^{2}}} \\
& =\frac{1}{\sqrt{1+|U(t)|^{2}}}+\frac{|U(t)|^{2}}{\sqrt{1+|U(t)|^{2}}} \\
& \leqq \frac{1}{\sqrt{1+|U(t)|^{2}}}+|X(t)| g(|X(t)|) \\
& <\sqrt{1+|U(0)|^{2}}+\int_{0}^{|X(t)|} g(|X(t)|) \mathrm{d} r \\
& \leqq \sqrt{1+|U(0)|^{2}}+\int_{0}^{|X(t)|} g(r) \mathrm{d} r
\end{aligned}
$$

since $g$ is decreasing,

$$
\begin{aligned}
& \leqq \sqrt{1+|U(0)|^{2}}+\|g\|_{1} \\
& \leqq \sqrt{1+|U(0)|^{2}}+\|g\|_{1}+\int_{|X(t)|}^{\left|X\left(t_{0}\right)\right|} g(r) \mathrm{d} r .
\end{aligned}
$$

We conclude that $(25)$ is satisfied with " $<$ " instead of " $\leqq$ " and thus is also satisfied for some right neighborhood $[t, t+\varepsilon[$ of $t$.

In all three cases it follows that $\left[t, t+\varepsilon\left[C S\right.\right.$. This shows that $S$ is open in $\left[t_{0}, t_{+}\right]$ and therefore $S=\left[t_{0}, t_{+}\right]$. In particular $(25)$ is satisfied for $t=t_{+}$:

$$
\begin{aligned}
\sqrt{1+\left|U\left(t_{+}\right)\right|^{2}} & \leqq \sqrt{1+|U(0)|^{2}}+\|g\|_{1}+\int_{\left|X\left(t_{+}\right)\right|}^{\left|X\left(t_{0}\right)\right|} g(r) \mathrm{d} r \\
& \leqq \sqrt{1+|U(0)|^{2}}+2\|g\|_{1} .
\end{aligned}
$$

Now either $T=t_{+}$, or we can use the simple case for $\left[t_{+}, T\right]$ to conclude that

$$
\sqrt{1+|U(T)|^{2}} \leqq \sqrt{1+\left|U\left(t_{+}\right)\right|^{2}}+\|g\|_{1} \leqq \sqrt{1+|U(0)|^{2}}+3\|g\|_{1},
$$

as claimed.

$5^{\circ}$ Now let us consider the case $c=0$. The preceding argument remains valid except for three places where we used that $X(t) \neq 0$ and $U(t) \neq 0$. The first place is line (26). Here we have to show that

$$
\left|U\left(t_{0}\right)\right|^{2} \leqq\left|U\left(t_{-}\right)\right|^{2}
$$

In the present situation we have that

$$
0=c=\left|U\left(t_{0}\right)\right|^{2}\left|X\left(t_{0}\right)\right|^{2}=\left|U\left(t_{-}\right)\right|^{2}\left|X\left(t_{-}\right)\right|^{2},
$$

and by the definition of $t_{0}$

$$
\left|X\left(t_{0}\right)\right|^{2} \geqq|X(t)|^{2}
$$

for all $t \in\left[t_{-}, t_{+}\right]$. If $t_{0}=t_{-}$, then (28) is true, so we assume $t_{0} \neq t_{-}$.

We claim that $\left|U\left(t_{0}\right)\right|=0$ which implies (28). In order to get a contradiction let 
$\left|U\left(t_{0}\right)\right| \neq 0$. Then $\left|X\left(t_{0}\right)\right|=0$ and therefore $|X(t)|=0$ on $\left[t_{-}, t_{0}\right]$. This implies $\frac{\mathrm{d}}{\mathrm{d} t} X(t)$ $=\hat{U}(t)=0$ on $\left[t_{-}, t_{0}\right]$ and therefore $0=U\left(t_{0}\right) \neq 0$.

The second place is after line (27). Here we have to show that

$$
\left|U\left(s_{+}\right)\right|^{2} \leqq\left|U\left(s_{-}\right)\right|^{2},
$$

but this is easy. We know that

$$
0=c=\left|U\left(s_{+}\right)\right|^{2}\left|X\left(s_{+}\right)\right|^{2}
$$

and

$$
\left|X\left(s_{+}\right)\right|>|X(s)| \geqq 0 .
$$

This shows that $\left|U\left(s_{+}\right)\right|^{2}=0$ which implies (29).

The third modification necessary is in the third case considered in $4^{\circ}$. If $U(t)=0=U(0)$, the old argument breaks down, but $\|g\|_{1} \neq 0$ and we still have strict inequality in (25).

(1.5) Theorem. (i) Every spherically symmetric solution $\left(f^{ \pm}, E, B \equiv 0\right)$ of $R V P S$ is also a solution of RVMS.

(ii) If $\left(f^{ \pm}, E, B\right)$ is a spherically symmetric solution of $R V M S$, then $B \equiv 0$ and $\left(f^{ \pm}, E, B\right)$ is also a solution of RVPS.

Proof. (i) The reader may easily convince herself that there is nothing to prove except (2) and (3). Because of the symmetry we have $\operatorname{curl} E=0$. This implies (3). This leaves only Eq. (2) which in this case $(B \equiv 0)$ is

$$
\partial_{t} E=-\gamma 4 \pi j(t, x) \text {. }
$$

We know that

$$
E(t, x)=\gamma \frac{x}{|x|^{3}} \int_{|y| \leqq|x|} \varrho(t, y) \mathrm{d} y=\gamma \frac{x}{|x|^{3}} \int_{|y| \leqq|x|}\left(f^{+}(t, y, w)-f^{-}(t, y, w)\right) \mathrm{d}(y, w) .
$$

We may compute $\partial_{t} E$ by differentiation under the integral sign, since $f^{ \pm}$is continuously differentiable and has compact support in $(y, w)$. Using Vlasov's equation we get that

$$
\begin{aligned}
\partial_{t}\left(f^{+}(t, x, u)-f^{-}(t, x, u)\right) & =-\hat{u} \partial_{x}\left(f^{+}-f^{-}\right)-(E+d \hat{u} \wedge B) \partial_{u}\left(f^{+}+f^{-}\right) \\
& =-\operatorname{div}_{x}\left(\hat{u}\left(f^{+}-f^{-}\right)\right)-\operatorname{div}_{u}\left((E+d \hat{u} \wedge B)\left(f^{+}+f^{-}\right)\right),
\end{aligned}
$$

cf. $[8,(1.3)]$. The integral of the second term vanishes and we conclude

$$
\begin{aligned}
\partial_{t} E(t, x) & =-\gamma \frac{x}{|x|^{3}} \int_{|y| \leqq|x|} \operatorname{div}_{y}\left(\hat{w}\left(f^{+}(t, y, w)-f^{-}(t, y, w)\right)\right) \mathrm{d}(y, w) \\
& =-\gamma \frac{x}{|x|^{3}} \int_{|y| \leqq|x|} \operatorname{div}_{y} j(t, y) \mathrm{d} y \\
& =-\gamma \frac{x}{|x|^{2}} 4 \pi\langle x, j(t, x)\rangle=-\gamma 4 \pi j(t, x)
\end{aligned}
$$

because of Gauss' Theorem and the spherical symmetry of $j(t, \cdot)$.

(ii) This follows with the same argument we used to derive Eq. (8). 


\section{Additional Remarks}

(i) We have once seen the coordinates $(a, b, c)$ from Lemma (1.2) in the astronomical literature of the 1950's or 1960's, but lost the reference.

(ii) The coordinate $c=x^{2} u^{2}-(x u)^{2}$ is the square of the angular momentum. Since this is a conserved quantity, this choice of coordinate system is particularly simple. Even so, we avoid non-cartesian coordinates. The only exception is the Integration Lemma (1.2) since this makes a later computation quite transparent.

(iii) There are two differences between the relativistic case $(d \neq 0)$ and the nonrelativistic case $(d=0)$. The first difference is that the non-relativistic "ODE Lemma" (cf. [7, (6.5)]) is much stronger than the relativistic version (1.4). This makes it necessary to use energy estimates in the relativistic case which can be avoided if $d=0$. Unfortunately the relativistic energy estimates are again not as good as the non-relativistic versions that work even for $\gamma=-1$. In the relativistic case with $\gamma=-1$ there exist counterexamples to global existence, cf. [2] and Sect. 3.

(iv) The main difficulty in the proof of Lemma (1.4) is that we make no assumption concerning the sign of $\widetilde{E}$. This enables us to apply the result in a situation where we do not know the sign of $\varrho$.

\section{On the Decay of a Symmetric Single-Species Plasma}

In this section we only consider the case that $f_{0}^{-}=0$ (and therefore $f^{-}=0$ ) and $\gamma=+1$. We drop the superscript " + ." Moreover, we only consider symmetric solutions. (Using the perturbation conjecture, however, we could also prove similar results in the case of "nearly symmetric" $f_{0}^{+}$with $f_{0}^{-}$"small".)

(2.1) Theorem. Assume that $f_{0} \geqq 0$ is continuously differentiable, symmetric and has compact support. Then there exist constants $C_{1}>0, C \geqq 0$ such that the $\operatorname{solution}(f, E)$ of $R V M S$ with $f(0, \cdot)=f_{0}$ satisfies

$$
|X(t, 0, x, u)|^{2} \geqq C_{1} \frac{x^{2} u^{2}-\langle x, u\rangle^{2}}{x^{2}} t^{2}
$$

for all $t \geqq 0,(x, u) \in \operatorname{supp} f_{0}$,

$$
|E(t, x)| \leqq C(1+t)^{-2}
$$

for all $t \geqq 0, x \in \mathbb{R}^{3}$,

$$
|\varrho(t, x)| \leqq C(1+t)^{-3}(1+\log (1+t))^{3}
$$

for all $t \geqq 0, x \in \mathbb{R}^{3}$,

$$
\left|\partial_{x} E(t, x)\right| \leqq C(1+t)^{-3}(1+\log (1+t))^{3}
$$

for all $t \geqq 0, x \in \mathbb{R}^{3}$.

Proof. (i) We consider the case $d=0$ first. Take any pair $(x, u) \in \mathbb{R}^{3} \times \mathbb{R}^{3}$. We abbreviate

$$
\begin{aligned}
& X(t):=X(t, 0, x, u), \\
& U(t):=U(t, 0, x, u) .
\end{aligned}
$$


By conservation of angular momentum

$$
|X(t)|^{2}|U(t)|^{2}-\langle X(t), U(t)\rangle^{2}=x^{2} u^{2}-\langle x, u\rangle^{2}=: c=\text { const }
$$

for all $t \geqq 0$. We assume $c>0$. (If $c=0$, the statement is trivial.) This shows that $X$ and $U$ have no zeroes. Moreover

$$
\begin{aligned}
& \frac{\mathrm{d}}{\mathrm{d} t}|X(t)|^{2}=2\langle X(t), U(t)\rangle, \\
& \frac{\mathrm{d}}{\mathrm{d} t}|U(t)|^{2}=2\langle U(t), E(t, X(t))\rangle=2\langle U(t), X(t)\rangle|X(t)|^{-3} \int_{|y| \leqq|x(t)|}^{\int} \varrho(t, y) \mathrm{d} y .
\end{aligned}
$$

Since $\varrho$ is nonnegative, this implies that $|U|$ is increasing (or decreasing, respectively) on an interval $I$ if $|X|$ is increasing (decreasing) on $I$. Differentiating (30) we get

$$
\left.\begin{array}{rl}
\frac{\mathrm{d}^{2}}{\mathrm{~d} t^{2}}|X(t)|^{2} & =2|U(t)|^{2}+2\langle X(t), E(t, X(t))\rangle \\
& =2|U(t)|^{2}+2|X(t)|^{-1} \int_{|y| \leqq|X(t)|} \varrho(t, y) \mathrm{d} y \geqq 2|U(t)|^{2}>0 .
\end{array}\right\}
$$

Therefore $X^{2}$ is strictly convex on $[0, \infty$ [. At most three cases are possible:

First case. $X^{2}$ is decreasing on $[0, \infty[$. Then

$$
\frac{\mathrm{d}^{2}}{\mathrm{~d} t^{2}}|X(t)|^{2} \geqq 2|U(t)|^{2} \geqq 2 \frac{c}{|X(t)|^{2}} \geqq 2 \frac{c}{x^{2}}>0 .
$$

This implies that $X^{2}$ is finally increasing, a contradiction that shows that this case is impossible.

Second case. $X^{2}$ is increasing on $\left[0, \infty\left[\right.\right.$. Then $U^{2}$ is increasing, too. We have

and

$$
\left.\frac{\mathrm{d}}{\mathrm{d} t}|X(t)|^{2}\right|_{t=0}=2\langle x, u\rangle \geqq 0
$$

for all $t \geqq 0$. Thus

$$
|U(t)|^{2} \geqq u^{2}
$$

$$
\frac{\mathrm{d}^{2}}{\mathrm{~d} t^{2}}|X(t)|^{2} \geqq 2|U(t)|^{2} \geqq 2 u^{2},
$$

and after integrating this twice we get

$$
|X(t)|^{2} \geqq x^{2}+2\langle x, u\rangle t+u^{2} t^{2} \geqq u^{2} t^{2} \geqq \frac{c}{x^{2}} t^{2} .
$$

Third case. (This is the interesting case.) There exists $t_{*}>0$ such that $X^{2}$ is decreasing on $\left[0, t_{*}\right]$ and increasing on $\left[t_{*}, \infty\left[\right.\right.$. We let $x_{*}=X\left(t_{*}\right), u_{*}=U\left(t_{*}\right)$ and note that

$$
\begin{aligned}
& x_{*}^{2}=\min _{t \geqq 0}|X(t)|^{2} \\
& u_{*}^{2}=\min _{t \geqq 0}|U(t)|^{2}
\end{aligned}
$$


and

$$
0=\left.\frac{\mathrm{d}}{\mathrm{d} t}|X(t)|^{2}\right|_{t=t_{*}}=2\left\langle x_{*}, u_{*}\right\rangle
$$

which in turn implies

$$
c=x_{*}^{2} u_{*}^{2} .
$$

As in the second case we conclude by integrating the second derivative

$$
|X(t)|^{2} \geqq x_{*}^{2}+\left(t-t_{*}\right)^{2} u_{*}^{2} .
$$

For later use we note that this implies for $t=0$,

$$
x^{2} \geqq x_{*}^{2}+t_{*}^{2} u_{*}^{2} .
$$

Because of the above estimate we have

$$
\frac{|X(t)|^{2}}{t^{2}} \geqq \frac{x_{*}^{2}+\left(t-t_{*}\right)^{2} u_{*}^{2}}{t^{2}} .
$$

Using freshperson calculus one can show that the minimum of the right-hand side over $t>0$ is

$$
\frac{c}{x_{*}^{2}+t_{*}^{2} u_{*}^{2}} \geqq \frac{c}{x^{2}} .
$$

(The last inequality follows from (32).) Thus

$$
|X(t)|^{2} \geqq \frac{c}{x^{2}} t^{2}
$$

as we have claimed (with $C_{1}=1$ ).

Now let $d>0$. A straightforward calculation gives

$$
\begin{aligned}
\frac{\mathrm{d}^{2}}{\mathrm{~d} t^{2}}|X(t)|^{2}= & 2\left(1+d^{2}|U(t)|^{2}\right)^{-1 / 2}|U(t)|^{2}+2\left(1+d^{2}|U(t)|^{2}\right)^{-3 / 2} \\
& \times\left(\left(1+d^{2}|U(t)|^{2}\right)|X(t)|^{-1}-d^{2}\langle X(t), U(t)\rangle^{2}|X(t)|^{-3}\right) \\
& \times \int_{|y| \leqq|X(t)|} \varrho(t, y) \mathrm{d} y \\
\geqq & 2\left(1+d^{2}|U(t)|^{2}\right)^{-1 / 2}|U(t)|^{2}
\end{aligned}
$$

Assume (finally!) that $(x, u) \in \operatorname{supp} f_{0}$.

We use the fact that $\operatorname{supp} f_{0}$ is bounded and estimate (18) to conclude that

$$
|U(t)| \leqq \text { const }
$$

uniformly for all $(x, u) \in \operatorname{supp} f_{0}$ and that the estimate ending on line (33) therefore implies that

$$
\frac{\mathrm{d}^{2}}{\mathrm{~d} t^{2}}|X(t)|^{2} \geqq 2 C_{1}|U(t)|^{2}
$$

with a positive constant $C_{1}$. 
This estimate now replaces (31) and we can proceed exactly as in the case $d=0$. (ii) Let $|x|=R$. Then

$$
\begin{aligned}
|E(t, x)| & =R^{-2} \int_{|y|^{2} \leqq R^{2}} \varrho(t, y) \mathrm{d} y=R^{-2} \int_{|y|^{2} \leqq R^{2}} f(t, y, w) \mathrm{d}(y, w) \\
& =R^{-2} \int_{|y|^{2} \leqq R^{2}} f_{0}(X(0, t, y, w), U(0, t, y, w)) \mathrm{d}(y, w) \\
& =R^{-2} \int_{|X(t, 0, y, w)|^{2} \leqq R^{2}} f_{0}(y, w) \mathrm{d}(y, w) .
\end{aligned}
$$

Using (i) we conclude that this is

$$
\begin{aligned}
& \leqq R^{-2} \\
& C_{1} \frac{y^{2} w^{2}-\langle y, w\rangle^{2}}{y^{2}} t^{2} \leqq R^{2} \\
& =R^{-2} 4 \pi^{2} f_{0}(y, w) \mathrm{d}(y, w) \\
& \operatorname{cic}_{1} t^{2} a^{-2} \leqq R^{2} \\
& f_{0}(a, b, c) \mathrm{d}(a, b, c) .
\end{aligned}
$$

Here we have defined

$$
f_{0}(y, w)=: \bar{f}_{0}\left(|y|,|y|^{-1}\langle y, w\rangle, y^{2} w^{2}-\langle y, w\rangle^{2}\right)
$$

and used the Integration Lemma (1.2). Since $f_{0}$ has compact support, the integration is over a set of the form

$$
0 \leqq a \leqq C, \quad|b| \leqq C, \quad 0 \leqq c \leqq C R^{2} t^{-2} .
$$

Since $f_{0}$ and therefore $\bar{f}_{0}$ is bounded, it follows that

$$
|E(t, x)| \leqq R^{-2} C R^{2} t^{-2}=C t^{-2} .
$$

Together with (21) this proves (ii).

(iii) Let $(x, u) \in \operatorname{supp} f(t, \cdot) ; t, \tau \geqq 0$ and abbreviate

$$
\begin{aligned}
& X=X(t, \tau, x, u), \\
& U=U(t, \tau, x, u), \\
& E=E(t, X(t, \tau, x, u)) .
\end{aligned}
$$

By straightforward calculation

$$
\begin{aligned}
\frac{\mathrm{d}}{\mathrm{d} t} X & =\hat{U} \\
\frac{\mathrm{d}^{2}}{\mathrm{~d} t^{2}} X & =\left(1+d^{2} U^{2}\right)^{-3 / 2}\left(\left(1+d^{2} U^{2}\right) E-d^{2}\langle U, E\rangle U\right) .
\end{aligned}
$$

This implies

$$
\left|\frac{\mathrm{d}^{2}}{\mathrm{~d} t^{2}} X\right| \leqq|E| \leqq C(1+t)^{-2}
$$

because of (i). Writing $X$ as its Taylor polynomial with integral remainder

$$
X=x+(t-\tau) \hat{u}+\int_{\tau}^{t} \int_{\tau}^{s} \frac{\mathrm{d}^{2}}{\mathrm{~d} \sigma^{2}} X(\sigma, \tau, x, u) \mathrm{d} \sigma \mathrm{d} s,
$$


we get the following estimate:

$$
\begin{aligned}
|X(0, t, x, u)| & \geqq|x-t \hat{u}|-\int_{0}^{t} \int_{s}^{t} C(1+\sigma)^{-2} \mathrm{~d} \sigma \mathrm{d} s \\
& =|x-t \hat{u}|-C \int_{0}^{t}\left((1+s)^{-1}-(1+t)^{-1}\right) \mathrm{d} s \\
& \geqq|x-t \hat{u}|-C \int_{0}^{t}(1+s)^{-1} \mathrm{~d} s \\
& =|x-t \hat{u}|-C \log (1+t)
\end{aligned}
$$

We have assumed that $f_{0}$ has compact support and so there exists an $R \geqq 0$ such that $f_{0}(y, w)=0$ if $|y| \geqq R$. Now

$$
\varrho(t, x)=\int f(t, x, u) d u=\int f_{0}(X(0, t, x, u), U(0, t, x, u)) \mathrm{d} u .
$$

The integration is over the set of all $u$ with $|X(0, t, x, u)| \leqq R$. But, because of the estimate ending on line (34), this set is contained in the set of all $u$ with

$$
|x-t \hat{u}|-C \log (1+t) \leqq R,
$$

or

$$
\left|\hat{u}-t^{-1} x\right| \leqq t^{-1}(R+C \log (1+t)) \text {. }
$$

Now let us look again at Eq. (35), the definition of $\varrho$. In the integral we substitute $v=\hat{u}$. Since $f(t, x, \cdot)$ lives on some bounded subset of $u$-space, uniformly for all $t \geqq 0, x \in \mathbb{R}^{3}$ (cf. (23)), we can estimate the determinant of the Jacobian with a constant. Now (36) implies that the $v$-integral is only over a ball of radius $\leqq t^{-1}(R+C \log (1+t))$ in 3-space. This shows that

$$
\varrho(t, x) \leqq C t^{-3}(1+\log (1+t))^{3},
$$

and taking (19) into account, we have proved (iii).

(iv) Follows from the next lemma, applied to $\psi=\tilde{\varrho}(t, \cdot)$.

(2.2) Lemma. Assume that $\psi:[0, \infty[\rightarrow \mathbb{R}$ is continuous, Hölder continuous at $x=0$ and bounded. Define

$$
K(x)= \begin{cases}\frac{x}{|x|^{3}} \int_{0}^{|x|} r^{2} \psi(r) \mathrm{d} r, & x \neq 0 \\ 0, & x=0 .\end{cases}
$$

Then $K$ is continuously differentiable on $\mathbb{R}^{3}$ and

Proof. If $x \neq 0$, we have

$$
\left|\frac{\partial K_{i}}{\partial x_{j}}(x)\right| \leqq 2\|\psi\|_{\infty} \text {. }
$$

$$
\frac{\partial K_{i}}{\partial x_{j}}=\left(\frac{\delta_{i j}}{|x|^{3}}-\frac{3 x_{i} x_{j}}{|x|^{5}}\right) \int_{0}^{|x|} r^{2} \psi(r) \mathrm{d} r+\frac{x_{i}}{|x|^{3}} \frac{x_{j}}{|x|}|x|^{2} \psi(|x|) .
$$

This is obviously continuous for $x \neq 0$. Moreover,

$$
\left|\frac{\partial K_{i}}{\partial x_{j}}\right| \leqq\left(\frac{3}{|x|^{3}} \int_{0}^{|x|} r^{2} \mathrm{~d} r\right)\|\psi\|_{\infty}+\|\psi\|_{\infty}=2\|\psi\|_{\infty} .
$$


Writing (37) as

$$
\frac{\partial K_{i}}{\partial x_{j}}=\delta_{i j} \frac{1}{|x|^{3}} \int_{0}^{|x|} r^{2} \psi(r) \mathrm{d} r+3 \frac{x_{i} x_{j}}{|x|^{2}} \frac{1}{|x|^{3}} \int_{0}^{|x|} r^{2}(\psi(|x|)-\psi(0)+\psi(0)-\psi(r)) \mathrm{d} r,
$$

we can easily see that

$$
\lim _{x \rightarrow 0} \frac{\partial K_{i}}{\partial x_{j}}=\frac{1}{3} \delta_{i j} \psi(0)
$$

Additional Remarks.

(i) The proof of Theorem (2.1) makes heavy use of the fact that $\varrho$ is nonnegative. It would be desirable to extend the argument to the case $f_{0}^{-} \neq 0$, in which $\varrho$ can assume both signs.

(ii) The constants in the statement of (2.1) depend on $d$. This makes sense, since for $d \rightarrow \infty$ the speed of light tends to zero. Thus all motion freezes and decay can no longer take place.

\section{The LaGrange-Jacobi Identity}

The following theorem is an analog of the LaGrange-Jacobi identity for the $N$-body problem (cf. [10]).

(3.1) Theorem. Assume that $\left(f^{+}, f^{-}, E, B\right)$ is a solution of $R V M S$ such that for every $T \geqq 0$ there exists a compact set $K_{0} \subset \mathbb{R}^{3}$ with

$$
\operatorname{supp} E(t, \cdot) \cup \operatorname{supp} B(t, \cdot) \subset K_{0}
$$

for all $t \in[0, T]$. Define for $t \geqq 0$,

$$
\begin{aligned}
I(t)= & \int x^{2}\left(1+d^{2} u^{2}\right)^{1 / 2}\left(f^{+}(t, x, u)+f^{-}(t, x, y)\right) \mathrm{d}(x, u) \\
& +\frac{d^{2}}{8 \pi \gamma} \int x^{2}\left(|E(t, x)|^{2}+|B(t, x)|^{2}\right) \mathrm{d} x .
\end{aligned}
$$

Then I is twice continuously differentiable and

$$
\begin{aligned}
& \dot{I}(t)=2 \int x u\left(f^{+}(t, x, u)+f^{-}(t, x, u)\right) \mathrm{d}(x, u)+\frac{d}{2 \pi \gamma} \int\langle x, E(t, x) \wedge B(t, x)\rangle \mathrm{d} x, \\
& \ddot{I}(t)=2 \int u \hat{u}\left(f^{+}(t, x, u)+f^{-}(t, x, u)\right) \mathrm{d}(x, u)+\frac{1}{4 \pi \gamma} \int\left(|E(t, x)|^{2}+|B(t, x)|^{2}\right) \mathrm{d} x .
\end{aligned}
$$

Proof. $1^{\circ}$ Because of our assumptions concerning the compact support of $f_{0}^{ \pm}$, $E(t, \cdot)$, and $B(t, \cdot)$ this is a straightforward exercise in the differentiation of integrals. The first term of $I$ is easily handled using the substitution (11) and the characteristic equations (10); for the second one we use Maxwell's equations.

$2^{\circ}$ In order to facilitate the logic of a later argument we first note that for every continuously differentiable function $K: \mathbb{R}^{3} \rightarrow \mathbb{R}^{3}$ with compact support we have

$$
\int\langle x,(\operatorname{curl} K(x)) \wedge K(x)\rangle \mathrm{d} x=\int\left(\frac{1}{2}|K(x)|^{2}-\langle K(x), x\rangle \operatorname{div} K(x)\right) \mathrm{d} x .
$$

This follows at once by integrating the elementary identity

$$
\operatorname{div}\left(K \wedge(K \wedge x)+\frac{1}{2}|K|^{2} x\right)=\langle K \wedge x, \operatorname{curl} K\rangle-\frac{1}{2}|K|^{2}+\langle K, x\rangle \operatorname{div} K .
$$


$3^{\circ}$ We now compute the first derivative of $I$ :

$$
\begin{aligned}
\dot{I}= & 2 \int\left(x u\left(f^{+}+f^{-}\right)+d^{2} x^{2} \hat{u} E\left(f^{+}-f^{-}\right)\right) \mathrm{d}(x, u) \\
& +\frac{d}{8 \pi \gamma} 2 \int x^{2}\left(\left\langle E, d^{-1} \operatorname{curl} B-4 \pi \gamma j\right\rangle-\left\langle B, d^{-1} \operatorname{curl} E\right\rangle\right) \mathrm{d} x \\
= & 2 \int x u\left(f^{+}+f^{-}\right) \mathrm{d}(x, u)+\frac{d}{4 \pi \gamma} \int x^{2}(\langle E, \operatorname{curl} B\rangle-\langle B, \operatorname{curl} E\rangle) \mathrm{d} x \\
= & 2 \int x u\left(f^{+}+f^{-}\right) \mathrm{d}(x, u)+\frac{d}{4 \pi \gamma} \int x^{2} \operatorname{div}(B \wedge E) \mathrm{d} x \\
= & 2 \int x u\left(f^{+}+f^{-}\right) \mathrm{d}(x, u)-\frac{d}{2 \pi \gamma} \int\langle x, B \wedge E) \mathrm{d} x,
\end{aligned}
$$

as claimed.

$4^{\circ}$ Similarly, using $2^{\circ}$, we obtain

$$
\begin{aligned}
\ddot{I}= & 2 \int\left(u \hat{u}\left(f^{+}+f^{-}\right)+\langle x, E+d \hat{u} \wedge B\rangle\left(f^{+}-f^{-}\right)\right) \mathrm{d}(x, u) \\
& +\frac{d}{2 \pi \gamma} \int\left\langle x,\left(d^{-1} \operatorname{curl} B-4 \pi \gamma j\right) \wedge B-E \wedge d^{-1} \operatorname{curl} E\right\rangle \mathrm{d} x \\
= & 2 \int u \hat{u}\left(f^{+}+f^{-}\right) \mathrm{d}(x, u)+2 \int\langle x, \varrho E+d j \wedge B\rangle \mathrm{d} x \\
& +\frac{1}{2 \pi \gamma} \int\langle x,(\operatorname{curl} B) \wedge B+(\operatorname{curl} E) \wedge E\rangle \mathrm{d} x-2 d \int\langle x, j \wedge B\rangle \mathrm{d} x \\
= & 2 \int u \hat{u}\left(f^{+}+f^{-}\right) \mathrm{d}(x, u)+2 \int\langle x, E\rangle \varrho \mathrm{d} x \\
& +\frac{1}{4 \pi \gamma} \int\left(|E|^{2}+|B|^{2}-2\langle x, E\rangle \operatorname{div} E\right) \mathrm{d} x \\
= & 2 \int u \hat{u}\left(f^{+}+f^{-}\right) \mathrm{d}(x, u)+\frac{1}{4 \pi \gamma} \int\left(|E|^{2}+|B|^{2}\right) \mathrm{d} x .
\end{aligned}
$$

Remarks. (i) The condition about the compact support of $E(t, \cdot)$ and $B(t, \cdot)$ is more restrictive than necessary. It could be replaced by growth conditions if the need would arise.

(ii) Examples of solutions of RVMS that satisfy the assumptions of the preceding theorem are the solutions for "nearly neutral" initial data of [3].

In the symmetric case we can discard the assumption about the compact support of $E(t, \cdot)(B \equiv 0$ anyway) if we slightly modify the definition of $I$ :

(3.2) Theorem. Assume that $\left(f^{+}, f^{-}, E\right)$ is a spherically symmetric solution of RVMS. Let

$$
\begin{aligned}
\widetilde{I}(t)= & \int x^{2}\left(1+d^{2} u^{2}\right)^{1 / 2}\left(f^{+}(t, x, u)+f^{-}(t, x, u)\right) \mathrm{d}(x, u) \\
& +\frac{d^{2}}{8 \pi \gamma} \int x^{2}\left(|E(t, x)|^{2}-|E(0, x)|^{2}\right) \mathrm{d} x .
\end{aligned}
$$

Then $\tilde{I}$ is twice continuously differentiable and

$$
\ddot{\widetilde{I}}(t)=2 \int u \hat{u}\left(f^{+}(t, x, u)+f^{-}(t, x, u)\right) \mathrm{d}(x, u)+\frac{1}{4 \pi \gamma} \int|E(t, x)|^{2} \mathrm{~d} x .
$$


Proof. This is exactly the same argument as in the preceding proof. The second integral in the definition of $\tilde{I}$ exists, since according to (17)

$$
E(t, x)=E(0, x)
$$

if $x$ is outside of a ball that contains the support of $\varrho(t, \cdot)$.

The LaGrange-Jacobi identity has some notable consequences:

(3.3) Theorem. Assume $\gamma=+1$. Then there exist no stationary solutions of RVMS that satisfy the assumptions of (3.1) or (3.2), apart from the zero solution.

Proof. For a stationary solution we would have $\ddot{I}=0$ (or $\ddot{\tilde{I}}=0$, respectively), but $\ddot{I}$ (or $\tilde{I}$ ) is strictly positive if $\gamma=+1$.

More quantitative information is gained if we use the conservation of the energy

$$
\begin{aligned}
H= & \int \frac{u^{2}}{\sqrt{1+d^{2} u^{2}}+1}\left(f^{+}(t, x, u)+f^{-}(t, x, u)\right) \mathrm{d}(x, u) \\
& +\frac{1}{8 \pi \gamma} \int\left(|E(t, x)|^{2}+|B(t, x)|^{2}\right) \mathrm{d} x .
\end{aligned}
$$

We note that

$$
\frac{u^{2}}{\sqrt{1+d^{2} u^{2}}+1}= \begin{cases}d^{-2}\left(\sqrt{1+d^{2} u^{2}}-1\right), & d>0 \\ \frac{1}{2} u^{2}, & d=0\end{cases}
$$

and

$$
u \hat{u}= \begin{cases}d^{-2}\left(\sqrt{1+d^{2} u^{2}}-\frac{1}{\sqrt{1+d^{2} u^{2}}}\right), & d>0 \\ u^{2}, & d=0 .\end{cases}
$$

This shows that

$$
\ddot{I} \geqq 2 H=\text { const }
$$

and, if $d>0$,

$$
\dddot{I} \leqq 2 H+2 d^{-2} \int\left(f^{+}(t, x, u)+f^{-}(t, x, u)\right) \mathrm{d}(x, u)=C=\text { const },
$$

the same estimates being true for $\ddot{\tilde{I}}$.

In the symmetric case we can get more precise information from the LaGrangeJacobi identity: Let us first assume that $\left(f^{+}, f^{-}, E\right)$ is a symmetric solution of RVMS such that $E(0, \cdot)$ (and therefore $E(t, \cdot)$ for all $t \geqq 0$ ) has compact support. Then

$$
\begin{aligned}
\int x^{2}|E|^{2} \mathrm{~d} x & =\int \frac{1}{x^{2}}\left(\int_{|y| \leqq|x|} \varrho(t, y) d y\right)^{2} \mathrm{~d} x \\
& =(4 \pi)^{3} \int_{0}^{\infty}\left(\int_{0}^{r} s^{2} \tilde{\varrho}(t, s) \mathrm{d} s\right)^{2} \mathrm{~d} r \\
& =-(4 \pi)^{3} \int_{0}^{\infty} r 2 r^{2} \tilde{\varrho}(t, r) \int_{0}^{r} s^{2} \tilde{\varrho}(t, s) \mathrm{d} s \mathrm{~d} r
\end{aligned}
$$


after partial integration, and we can bound this by

$$
\begin{aligned}
& \leqq(4 \pi)^{2} \cdot 2 \int_{0}^{\infty} r^{3}|\varrho(t, r)| \mathrm{d} r \cdot \int|\varrho(t, x)| \mathrm{d} x \\
& =8 \pi \int|x||\varrho(t, x)| \mathrm{d} x \cdot \int|\varrho(t, x)| \mathrm{d} x \\
& \leqq 8 \pi\left(\int x^{2}|\varrho(t, x)| \mathrm{d} x\right)^{1 / 2}\left(\int|\varrho(t, x)| \mathrm{d} x\right)^{3 / 2} \\
& =C\left(\int x^{2}|\varrho(t, x)| \mathrm{d} x\right)^{1 / 2} \\
& \leqq C\left(\int x^{2}\left(1+d^{2} u^{2}\right)^{1 / 2}\left(f^{+}+f^{-}\right) \mathrm{d}(x, u)\right)^{1 / 2}
\end{aligned}
$$

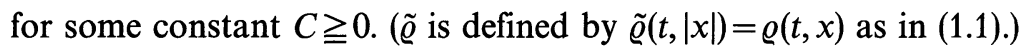
that

If $E(0, \cdot)$ does not necessarily have compact support, the same argument shows

$$
\left|\int x^{2}\left(|E(t, x)|^{2}-|E(0, x)|^{2}\right) \mathrm{d} x\right| \leqq C\left(1+\left(\int x^{2}\left(1+d^{2} u^{2}\right)^{1 / 2}\left(f^{+}+f^{-}\right) \mathrm{d}(x, u)\right)^{1 / 2}\right) .
$$

Thus for a symmetric solution $\left(f^{+}, f^{-}, E\right)$ of RVMS the second integral in the definition of $\widetilde{I}(t)$ is essentially dominated by the square root of the first one. This leads to the following

(3.4) Theorem. Assume that $\left(f^{+}, f^{-}, E\right)$ is a symmetric solution of $R V M S$. If the total energy $H$ is positive, there exists a positive constant $C$, such that

$$
\begin{gathered}
\int\left(\left|X^{+}(t, 0, x, u)\right|^{2} f_{0}^{+}(x, u)+\left|X^{-}(t, 0, x, u)\right|^{2} f_{0}^{-}(x, u)\right) \mathrm{d}(x, u) \\
=\int|x|^{2}\left(f^{+}(t, x, u)+f^{-}(t, x, u)\right) \mathrm{d}(x, u) \geqq C t^{2} .
\end{gathered}
$$

Proof. By (23)

$$
\int|x|^{2}\left(f^{+}+f^{-}\right) \mathrm{d}(x, u) \geqq C \int|x|^{2}\left(1+d^{2} u^{2}\right)^{1 / 2}\left(f^{+}+f^{-}\right) \mathrm{d}(x, u),
$$

and by (38)

$$
\widetilde{I}(t) \geqq C t^{2}
$$

for large $t$.

Because of (40), this implies that

$$
\int|x|^{2}\left(1+d^{2} u^{2}\right)^{1 / 2}\left(f^{+}+f^{-}\right) \mathrm{d}(x, u) \geqq C t^{2}
$$

for large $t$, and therefore also

$$
\int|x|^{2}\left(f^{+}+f^{-}\right) \mathrm{d}(x, u) \geqq C t^{2}
$$

for large $t$. However, since $\int|x|^{2}\left(f^{+}+f^{-}\right) \mathrm{d}(x, u)$ is a positive continuous function of $t$, such an estimate is also true for small $t$.

Remark. The essential ingredient of the proof of Theorem (2.1) was the growth estimate (i):

$$
|X(t, 0, x, u)|^{2} \geqq C_{1} c|x|^{-2} t^{2} .
$$

Whereas we could not prove this for a system with $f_{0}^{-} \neq 0$, the preceding theorem shows that at least some weaker integral version of this estimate remains true. This gives rise to the hope that the decay results of Sect. 2 might also be valid for $f_{0}^{-} \neq 0$. 
We end with a non-existence result:

(3.5) Theorem. If $\gamma=-1$ and $d>0$, there exists no spherically symmetric global solution $\left(f^{+}, f^{-}, E\right)$ of $R V M S$ with

$$
d^{-2} \int \sqrt{1+d^{2} u^{2}}\left(f_{0}^{+}+f_{0}^{-}\right) \mathrm{d}(x, u)-\frac{1}{8 \pi} \int\left|E_{0}(x)\right|^{2} \mathrm{~d} x<0 .
$$

Proof. This condition means exactly that the constant $C$ in (39) is negative. Thus we would have

$$
\lim _{t \rightarrow \infty} \widetilde{I}(t)=-\infty .
$$

On the other hand, we conclude from (40) that $\widetilde{I}(t)$ is bounded below.

\section{Additional Remarks}

(i) Theorem (3.5) implies that for $\gamma=-1, d>0$ there exist counter-examples to global existence, cf. [2].

(ii) A generalization of the definition of $\tilde{I}$ to the non-symmetric case would be welcome.

\section{References}

1. Di Perna, R.J., Lions, P.L.: Global weak solutions of Vlasov-Maxwell systems. Preprint 1988

2. Glassey, R.T., Schaeffer, J.: On symmetric solutions of the relativistic Vlasov-Poisson system. Commun. Math. Phys. 101, 459-473 (1985)

3. Glassey, R.T., Schaeffer, J.: Global existence for the relativistic Vlasov-Maxwell system with nearly neutral initial data. Commun. Math. Phys. 119, 353-384 (1988)

4. Glassey, R.T., Strauss, W.A.: Singularity formation in a collisionless plasma could occur only at high velocities. Arch. Rat. Mech. Anal. 92, 59-90 (1986)

5. Glassey, R.T., Strauss, W.A.: Absence of shocks in an initially dilute collisionless plasma. Commun. Math. Phys. 113, 191-208 (1987)

6. Horst, E.: On the classical solutions of the initial value problem for the unmodified non-linear Vlasov equation I. Math. Meth. Appl. Sci. 3, 229-248 (1981)

7. Horst, E.: On the classical solutions of the initial value problem for the unmodified non-linear Vlasov equation II. Math. Meth. Appl. Sci. 4, 19-32 (1982)

8. Horst, E.: Global solutions of the relativistic Vlasov-Maxwell system of plasma physics. To appear in Dissertationes Mathematicae

9. Horst, E., Hunze, R.: Weak solutions of the initial value problem for the unmodified nonlinear Vlasov equation. Math. Meth. Appl. Sci. 6, 262-279 (1984)

10. Khil'mi, G.F.: Qualitative methods in the many body problem. New York: Gordon and Breach 1961

11. Rein, G.: Forthcoming Ph. D. dissertation

12. Schaeffer, J.: Global existence for the Poisson-Vlasov system with nearly symmetric data. J. Differ. Eqs. 69, 111-148 (1987)

Communicated by J. L. Lebowitz 
